\title{
Automatic object segmentation using perceptual grouping of regions with contextual constraints
}

\begin{abstract}
Image segmentation is still considered a very challenging subject despite years of research effort poured into the field. The problem is exacerbated when there is need for specific object detection. Since objects can be visually non-homogeneous, techniques that attempt to segment images into visually uniform regions using only the bottom-up cues, tend to fail. We propose a novel two-step model that incorporates both bottom-up information and top-down object constraints. Firstly, a set of uniform regions are generated using an extension of contour detection, seeded region growing, and graph-based methods. The second step applies co-occurrence constraints on the image regions in order to perceptually group regions into objects. This unsupervised segmentation process models each object using higher-level knowledge in the form of visual co-occurrences of its constituent parts. Experiments on the horse and ImageCLEF databases show that the proposed technique performs comparably well with existing state-of-the-art techniques.
\end{abstract}

Keyword: Automatic image segmentation; Contextual relationships; Graph-based segmentation; Object segmentation 